Palliativmedizin

\title{
EBM um acht Ziffern erweitert
}

— Die Palliativversorgung soll flächendeckend etabliert werden, so lautete der Auftrag aus dem Hospiz- und Palliativgesetz, das Ende 2015 verabschiedet wurde. Den zweiten entscheidenden Schritt dafür haben KBV und Kassen nun getan: Sie haben sich im Bewertungsausschuss darauf geeinigt, den EBM um acht neue Gebührenordnungspositionen (GOP) zu ergänzen, diese werden im neuen Abschnitt 37.3 gebündelt und - zunächst begrenzt auf zwei Jahre extrabudgetär vergütet.

Die neuen GOP sind für Haus- und Fachärzte abrechenbar und umfassen neben der palliativmedizinischen Ersterhebung und Koordination der Versorgung etwa auch Hausbesuche und Rufbereitschaft. Allerdings benötigen Ärzte für einen Teil der Leistungen eine Genehmigung der KV. Die neuen Leistungen im Überblick.

GOP, für die eine KV-Genehmigung vorliegen muss:

- 37300 - Palliativmedizinische Ersterhebung (392 Punkte, 41,28 Euro): Der Arzt muss anhand eines Assessments den individuellen palliativen Bedarf des Patienten ermitteln und einen entsprechenden Behandlungsplan erstellen oder diesen aktualisieren. Einmal im Krankheitsfall ansetzbar.

\section{- 37302 - Zuschlag für den koordinie-} renden Arzt (275 Punkte; 28,96 Euro): Es geht vor allem um die Gestaltung der $\mathrm{Zu}$ sammenarbeit mit Palliativdiensten, SAPV-Teams und Hospizdiensten. Der Zuschlag wird zur Versicherten- oder Grundpauschale gezahlt.

\section{_ 37317 - Zuschlag Erreichbarkeit/Be-} suchsbereitschaft (1425 Punkte, 150,05 Euro): Für die telefonische Erreichbarkeit und Besuchsbereitschaft außerhalb der Sprechstundenzeiten erhalten Ärzte eine einmalige Vergütung von 150 Euro (einmal im Krankheitsfall). Die Zeiten, wann der Arzt erreichbar ist, stimmt er mit den Patienten, den Angehörigen oder den Pflegekräften ab.

37318 - Telefonische Beratung (213 Punkte, 22,43 Euro): Auch

Neue Vergütung in der Palliativmedizin versprechen die acht neuen GOP.
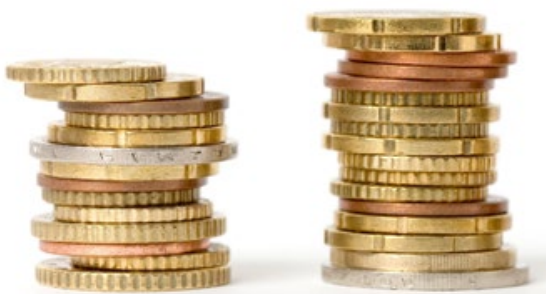

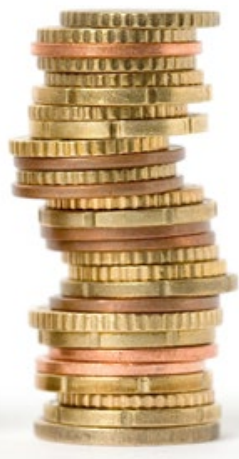
gepersonal, Angehörigen oder dem ärztlichen Bereitschaftsdienst außerhalb der Sprechstundenzeiten werden vergütet. Das Gespräch muss mindestens fünf Minuten dauern und die Leistung geht ins Tagesund Quartalsprofil ein. Höchstens sieben Mal pro Fall berechnungsfähig. längere Telefonate mit Pfle-
Ohne KV-Genehmigung abrechenbar:

_ 37305 - Zuschlag zum Hausbesuch (124 Punkte/ 13,05 Euro): Erfolgt die palliativmedizinische Betreuung des Patienten zu Hause, im Pflegeheim oder Hospiz, können Ärzte als Zuschlag zu den GOP 01410 und 01413 je vollendete 15 Minuten knapp 13 Euro berechnen. Die Leistung geht ebenfalls ins Tages- und Quartalsprofil ein und darf pro Behandlungstag bis zu sechs Mal also für Besuche bis maximal 1,5 Stunden angesetzt werden.

- 37306 - Zuschlag zu einem dringenden Hausbesuch (124 Punkte/13,06 Euro): kann im Unterschied zu 37305 nur einmal pro Besuch berechnet werden. Gilt nicht für Besuche im Rahmen des Not(fall)dienstes, der Notfallversorgung oder dringender Visiten auf der Belegstation.

- 37320 - Fallkonferenz gemäß Anlage 30 BMV-Ä (64 Punkte/ 6,74 Euro): Diese GOP gibt es für die patientenorientierte Fallbesprechung unter der Beteiligung der notwendigen ärztlichen Fachdisziplinen und/oder weiterer komplementärer Berufe sowie mit Pflegekräften beziehungsweise Angehörigen. Die Leistung kann fünf Mal im Krankheitsfall angesetzt werden und gilt auch bei einer Konferenz per Telefon.

Außerdem wird der EBM noch um eine Konsiliarärztliche Pauschale ergänzt, die GOP 37317 (106 Punkte/ 11,16 Euro). Diese Leistung steht nur konsiliarisch tätigen Ärzten mit der Zusatzweiterbildung Palliativmedizin zur Verfügung - und zwar dann, wenn sie von einem Haus- oder Facharzt wegen einer speziellen Fragestellung zu Rate gezogen werden, aber kein direkter Arzt-Patienten-Kontakt zustande kommt.

(reh)

Subklinische Hypothyreose

\section{L-Thyroxin dämpft Migräne}

— Bei Migränepatienten mit ausgeprägter Hypothyreose machen Ärzte oft gute Erfahrungen mit einer Levothyroxin-Behandlung. Ob eine solche Therapie auch Patienten mit subklinischer Hypothyreose nützt, haben Anastasie Bougea et al., Universität Athen, Griechenland, geprüft. Sie verabreichten das Medikament 45
Migränepatienten ohne Aura, bei denen TSH-Werte über 4,5 $\mathrm{mU} / \mathrm{l}$ bei normalen T4-Werten gemessen wurde. Die Patienten bekamen zwei Monate lang 50-100 mg Levothyroxin pro Tag. Dabei ging die Schwere der Migräne auf einer 10-PunkteAnalogskala von 6,5 vor auf 1,2 Punkte nach der Behandlung zurück, die Zahl der monatlichen Migränetage sank von 14,7 auf 1,9. Beide Unterschiede waren statistisch signifikant. Die Studie liefere erste Hinweise, dass auch bei subklinischer Hypothyreose eine L-Thyroxin-Behandlung die Migränesymptome deutlich lindern könne, sagte Bougea auf dem europäischen Neurologenkongress in Amsterdam. Solche Hinweise müssten nun natürlich placebokontrolliert geprüft werden.

(mut) 